\title{
A Comprehensive Review of Significant Researches on Face Recognition Based on Various Conditions
}

\author{
S. Muruganantham, Member IACSIT and Dr. T. Jebarajan
}

\begin{abstract}
In current decades face recognition has acknowledged significant attention from both research communities and the market, however still remained very exciting in real applications. The assignment of face recognition has been dynamically researched in current ages. This paper offers an up-to-date evaluation of major human face recognition research. We first present an summary of face recognition and its applications. Then, a literature review of the predominantly used face recognition techniques is offered. Clarification and restrictions of face databases which are used to test the performance of these face recognition algorithms are given. The most important factors distressing the face recognition system is pose illumination, identity, occlusion and expression. This paper struggles on the papers with these factors. Here we project a vital assessment of the current researches associated with the face recognition process. In this paper, we present a wide review of major researches on face recognition process based on various conditions. In addition, we present a summarizing description of Face recognition process along with the techniques connected with the various factors that affects the face recognition process.
\end{abstract}

Index Terms - Image processing, biometrics, face recognition, face detection, pose, illumination, occlusion, expression.

\section{INTRODUCTION}

A short time ago, the use of biometrics has improved to a large extent in personal security and/or access control applications. Biometrics is a technology, which is likely to replace out dated certification methods that are easily stolen, elapsed and copied. Fingerprints, face, iris, and voiceprints are normally used biometric features [1]. Among these features, face offers a more direct, friendly and convenient documentation method. It is more conventional to users compared to other discrete identification methods of biometric features. Thus, face recognition shows an important role in biometrics. Face recognition, an effective biometric method [2], has varied applications particularly as an identification solution which can meet the desperate needs in security areas. It includes image processing, pattern recognition, intelligent Learning and so on [3]. Face recognition technologies have been developed considerably during the past few years, making it an effective tool for automatic entree control and video observation.

Face recognition, a kind of biometric credentials,

Manuscript received September 27, 2011; revised October 12, 2011.

Mr. S. Muruganantham is with MCA Department, S. T. Hindu College, Nagercoil, Kanyakumari District, Tamil Nadu, India (e-mail: smuru1970@gmail.com).

Dr. T.Jebarajan is with Kings College of Engineering, Chennai, India (e-mail: tjebaraj1970@gmail.com). examined in numerous fields such as computer vision, image processing, and pattern recognition is a usual and straight biometric method [4], [5]. Automated methods that use facial features as essential elements of discrepancy to determine identity are involved in the process of facial recognition [6]. Automatic face recognition as a means of human identification has been strongly studied for more than thirty years. The achievement of face recognition routine degrades with pose and lighting variations, although the recognition performance has been developed significantly under frontal pose and optimal lighting conditions [7]. Criminal Identification, security system, image and film processing are some of the profitable applications of face recognition [8]. Face recognition involves classification of extremely confusing multi-dimensional input signals, and matching them with the known 'signals' and it is an extremely problematical type of pattern recognition.

An excessively large numbers of training specimens are required for classifying a array with high dimensions. This is recognized as the 'Curse of Dimensionality'. Persons are generally identify by their face, and automatic face recognition is now possible because of the growths made in the computing capability over the past few years. Information security, law implementation and investigation, smart cards, access control are some of the zones that have potential applications for Face Recognition [9]-[11]. Since faces of varied persons contribute to global shape features, whereas face images of a single person is exposed to significant differences, which might overcome the measured inter-person variations, face recognition appears to have aspiring challenges. The facts that assimilate facial expressions, illumination conditions, pose, presence or absence of eyeglasses and facial hair cause such lack of correspondence [12], [13]. Output, simplicity, and non-invasiveness are dynamic advantages of face recognition as a biometric.

A subsist face image is captured and compared with the stored images by the Face Recognition system. A face is mainly a 3D object among casual background objects lighted by varied lighting sources from several orientations. For the reason that, when a face is projected in contradiction of a 2-D image its appearance diverges remarkably [14]. Main changes in 2-D appearance are also initiated by diverse pose angles. Competence of imagining out characteristics in spite of such changes in appearance that a face can have in a setting is necessary for robust face recognition. At the same time common image acquirement problems for example noise, video camera distortion, and image resolution must not disturb the working of the system [15]. Certain facial features vibrant to facial reading tasks, for example face recognition 
[16], facial expression analysis are eyes, eyebrows, nose and mouth, and their spatial positions and [17] face animation [18]. However, variation caused by factors such as occlusion, illumination, expression, pose, accessories and aged produces a presentation drop in all face recognition algorithms [19].

A face recognition system should handle the above mentioned usual constraints successfully by taking them into attention to a large scope. Well-organized and in effect characterization of faces is necessary to achieve this [20]. As per matter of fact, most of the time intra-individual variability of face images even higher than inter-individual variability results due to these factors [21]. The first step of face recognition is the proof of identity of an effective method to decrease the dimensionality. The Feature Based (Geometric) and Temple Based (Photometric) are the two types of face recognition.

Depending on the direction of the lighting and fixed pose, varied distinct shadows and secularities are made by each of these components. Direction of lighting can be considered and the illumination changes can be offset using such features [22]. Parameters for instance head pose and lighting influence the gray level or color values delivered to the recognition system that recognizes the face from images. The major challenge for face recognition [23] is the variations beyond the dissimilarity between different person's images that may be produced by the variations in pose and illumination. Usually, dealing with pose variation is more complex compared to illumination variation. When the variation in light is larger [24], it is not stress-free for the system to recognize the individuals. If the $3 \mathrm{D}$ shape is recognized, the pose and illumination invariant face recognition can be gifted effortlessly, because the pose and illumination changes are all connected with the face 3D structure.

Even for the same person, large variance of presence is caused by pose variation. The variance is often more prominent than that produced by different persons in the same pose [25]. Therefore the humiliation of conventional appearance-based techniques for instance Eigen face etc., degrades strictly when non frontal searches match beside the registered frontal faces [26]. Among the several methods offered to address the pose problem the widely used method are view-based methods [27]. Diverse appearance of the 3D shape of human faces under lighting from diverse direction is the central cause for difficulties associated with Illumination variation for facial image. Principal components analysis [28], discrete wavelets transform [29], and discrete cosine transforms [30] and Gabor wavelet-based representation [31] are some numerous methods for representing face images that have been proposed in the literature. But the achievement of these methods is not bright since they have got two important disadvantages. The first one is high computational complexity and the second one is the exceptionally large memory required for storing features [32, 33]. Little effort has been taken to deal with the problem of unified variations of pose and illumination, though numerous algorithms have been recommended for face recognition from fixed viewpoints [9].

Here in this paper, we present a wide analysis of important seeks on Face Recognition centred on various conditions along with its treating and analysis techniques. A summary of different face recognition systems hired in various prevailing techniques are shown. Based on the modelling necessities of a Face Recognition system, we study, categorize and go over the existing modelling methodologies. Moreover, we propose a brief description about Face Recognition based various factors that affecting the recognition process. The key factors that affecting the face recognition process are pose illumination, occlusion, identity and expressions. In our research, we can professionally identify the face based on the factors pose, illumination and occlusion. These factors can be noticed effectively by various methods. The other details are as follows: Section 2 describes the Face recognition process and the challenges in the face recognition process are explained in Section 3. A general review on the study of significant research methods in Face Recognition process is also provided in section 3. Section 4 ends with the conclusion.

\section{FACE RECOGNITION}

Face detection and recognition are the difficult complications in computer vision area. This is the intention why this field receives a vast consideration in medical field and research communities together with biometric, pattern recognition and computer vision communities [13, 9, 34, 35]. For several applications, the act of face recognition systems in controlled environments has now reached a adequate level; but, still there are many challenges posed by uncontrolled environments. Certain challenges are posed by the problems caused by variations in illumination, face pose, expression, Identity and occlusion etc. [36]. All existing face recognition techniques can be classified into four types based on the way they signify the face. They are

1. Appearance based which uses all-inclusive texture features.

2. Model based which works shape and texture of the face, along with 3D depth information.

3. Pattern based face recognition.

4. Techniques using Neural Networks

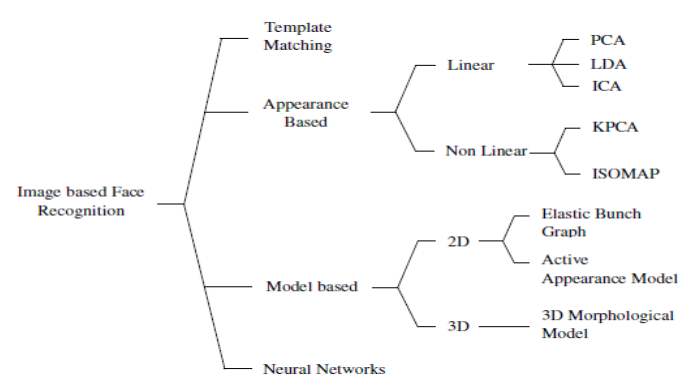

Fig. 1. Classification of face recognition methods

Generally, a face recognition system consist of three key elements:

1. Face detection and normalization;

2. Feature extraction and discriminant analysis;

3. Identification and/or verification.

The strength of face recognition may possibly improve by handling the variations in these elements. Feature extraction 
is a very important step for face recognition. Several methods need exact locations of key facial features such as eyes, nose, and mouth to stabilize the faces.

\section{Challenges of FACE Recognition System}

Though face recognition have been a grown up research area, however, there still remain many problems that must be overcome to develop a robust face recognition system that works well under various circumstances such as illumination, pose, occlusion, pose and illumination, pose and occlusion, illumination and occlusion and lastly illumination and pose and occlusion as shown in fig. 2 . The results reveal that all the recognition techniques were successful on large face databases recorded in well controlled environments. But under uncontrolled environments their performance gets deteriorated mainly due to variations on pose, illumination and occlusions. Such variations have proven to be one of the biggest problems of face recognition systems.

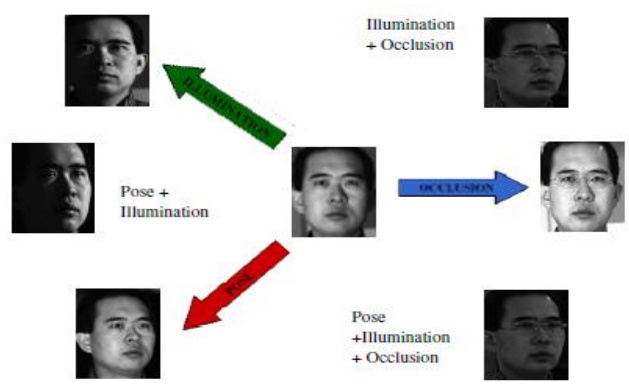

Fig. 2. Various challenges of face recognition system

Since we are representing a review paper on pose-illumination-occlusion invariant face recognition systems. So it is very essential to head in the direction of knowing what basic approaches had been adopted to deal with pose-illumination-occlusion invariant face recognition system. So to tackle this problem, the face with different poses and occlusions is recognized under diverse lighting conditions by our proposed system. The rest of the paper is structured as follows:

\section{A. Review Based on Pose}

Face recognition is a progressively popular biometric proof of identity technique, currently being implemented for security and law enforcement. It outrivals in these applications, as its submissive and non-intrusive nature allows continuous monitoring, while not disturbing persons. Even though the fact that face recognition has developed into an wide research field, around numerous major challenges prevailing, including handling changes in lighting, expression, facial hair and facial pose. Facial pose is a mostly difficult challenge, and is still an open research problem [37]. A number of pose invariant face recognition methodologies have been recommended in the past years. Fig. 3 represents some of the pose variations in FERET database.

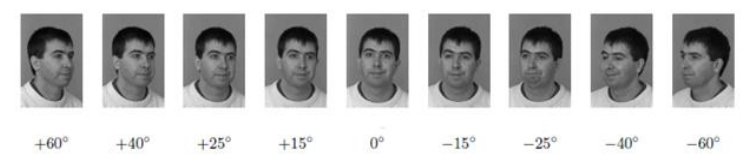

Fig. 3. Pose variation in FERET database
Tae-Kyun Kim and Josef Kittler [38] have suggested that the problem of pose-invariant face recognition centred on a single model image. To manage with novel view face images, a model of the effect of pose changes on face appearance must be available. Face images at a random pose can be mapped to a orientation pose by the model yielding view-invariant representation. Such a model usually be dependent on dense correspondences of different view face images, which remained difficult to establish in training. Errors in the correspondences totally damage the accuracy of any recognizer. They expected only the nominal possible set of correspondences, given by the corresponding eye positions. They examine a number of approaches to pose-invariant face recognition developing such a minimal set of facial features correspondences. Four different techniques were offered as pose-invariant face recognition "experts" and combined in a single framework of expert fusion. Each expert clearly or discreetly recognizes the three sequential functions jointly required to capture the nonlinear manifolds of face pose changes: representation, view transformation, and class discriminative feature extraction.

Hui-Fuang $\mathrm{Ng}$ [39] proposed that the Face recognition security systems had become essential for many submissions such as automatic access control and video surveillance. Most face recognition security systems required right frontal views of a person, and those systems was failed if the person to be predictable does not face the camera correctly. In their paper, they offer a novel approach for robust pose-invariant face recognition. Their recognition system was indifferent to viewing information and it requires only one sample view per person. Their method make use of the comparisons of a face image against a set of faces from a training set at the same view to inaugurate pose-invariant representations of a person in different poses.

Hyung-Soo Lee and Daijin Kim [1] have offered to recognize human faces were one of the most important areas of research in biometrics. Extreme change of facial pose was a big challenge for its useful application. Their papers propose generating frontal view face image using linear transformation in feature space for face recognition. They extract features from a posed face image using the kernel PCA. After that, they make over the posed face image into its equivalent frontal face image using the transformation matrix determined by learning. Then, the generated frontal face image was recognized by three different intolerance methods such as LDA, NDA, or GDA.

Eidenberger [40] have described that novel algorithm for the recognition of faces from image samples. Their algorithm used the Kalman filter to identify considerable facial character. Kalman faces were condensed visual models that represent the invariant extent of face classes. They utilize the Kalman faces approach on the UMIST database, a collection of face images that were record under varying camera angles. Kalman faces show strength against invisible facial character and break the classic Eigen faces approach in terms of recognition performance and algorithm speed. Their papers discuss Kalman faces extraction, application; tunable parameters, experimental results and related work on Kalman filter application in face recognition.

Ting Shan et al. [36] have described that majority face 
recognition systems only work well under quite constrain environment. In particular, the illumination conditions, facial expressions and head pose must be strongly embarrassed for good recognition performance. In 2004, they projected a new face recognition algorithm, Adaptive Principal Component Analysis (APCA) which performs well against both lighting variation and expression change. However like other eigenface-derived face recognition algorithms, APCA just performs well with frontal face images. They developed a face model and a rotation model which can be used to take facial features and create realistic frontal face images when given a single novel face image. They use a Viola-Jones based face detector to detect the face in real-time and thus solve the initialization problem for our Active Appearance Model search.

Hongzhou Zhang et al. [3] have projected that face recognition has varied applications particularly as an identification solution which can meet the lament needs in security areas. Pose problem was a big challenge applying that technology under real world conditions. Appearance based approach was proposed. Face recognition was implemented by reconstructing frontal view features using linear transformation

Xiujuan Chai et al. [41] have described that the difference of facial appearance due to the viewpoint (pose) degrades face recognition systems greatly, which was one of the bottleneck in face recognition. Their paper proposes a simple, but capable, novel locally linear regression (LLR) method, which generates the virtual frontal view from a given non-frontal face image. They first justify the basic theory of the paper that there exists an estimated linear mapping between a non-frontal face image and its frontal counterpart. Then, by formulating the assessment of the linear mapping as a prediction problem, we present the regression-based solution, i.e., worldwide linear regression. To improve the prediction accuracy in the case of coarse alignment, LLR was further proposed. In LLR, they first performed dense sampling in the non-frontal face image to obtain many overlapped local patches.

Richa Singh et al. [42] have described that the Mosaicing entails the consolidation of information represented by several images through the function of a registration and blending procedure. They described a face mosaicing system that generates a multiple face image during enrolment based on the proof provided by frontal and semi profile face images of an individual. Face mosaicing obviates the need to store multiple face templates indicating multiple poses of a user's face image. In their system, the side profile images were aligned with the frontal image using a hierarchical registration algorithm that exploits locality properties to determine the transformation relating the two images. Multi resolution splining was then used to merge the side profiles with the frontal image, thus generating a composite face image of the user.

Hasan Demirel and Gholamreza Anbarjafari [43] have proposed a new and high performance pose invariant face recognition system based on the Probability Distribution Functions (PDF) of pixels in different color channels is anticipated. The PDFs of the poised and segmented face images were used as geometric feature vectors for the recognition of faces by minimizing the Kullback-Leibler distance (KLD) between the PDF of a given face and the PDFs of faces in the database. Feature vector fusion (FVF) and majority voting (MV) methods have been engaged to combine feature vectors obtained from different color channels in $\mathrm{HSI}$ and $\mathrm{YCbCr}$ color spaces to recover the recognition performance.

Simon J.D. Prince and James H. Elder [44] have projected that face recognition algorithms perform very erratically when the pose of the probe face was different from the stored face: typical feature vectors vary more with pose than with character. They propose a generative model that creates a one-to-many mapping from an idealized "identity" space to the observed data space. In their identity space, the representation for each individual does not vary with pose. The measured feature vector was generated by a pose contingent linear transformation of the identity vector in the presence of noise. They term their model "tied" factor analysis. The selection of linear transformation (factors) depends on the pose, except the loadings are constant (tied) for a given individual. Their algorithm estimates the linear transformations and the noise parameters using training data. They proposed a probabilistic distance metric which allows a full subsequent over possible matches to be established. They introduced a novel feature extraction process and investigate recognition performance using the FERET database.

\section{B. Review Based on Illumination}

The illumination variation problem is one of the eminent problems in face recognition in uncontrolled environment. Designate more specific, the varying direction and energy distribution of the ambient illumination, self-possessed with the 3D structure of the human face, can lead to most important differences in the shading and shadows on the face. Such variations in the face appearance can be much larger than thevariation caused by personal identity [26]. The variations of both global face appearance and local facial features too cause problems for automatic face detection/ localisation, which is the necessity for the consequent face recognition stage. And so the situation is even inferior for a fully automatic face recognition system. Additionally, in a practical application environment, the illumination variation is always combined with other problems such as pose variation and expression variation, which increase the difficulty of the automatic face recognition problem. A number of illumination invariant face recognition approaches have been suggested in the past years. Fig.4 represents some of the images in Yale database $\mathrm{B}$ based on various illumination conditions.

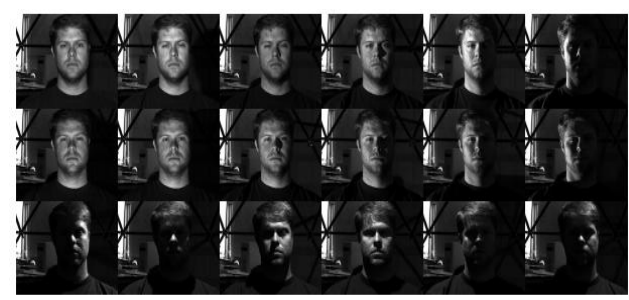

Fig. 4. Illumination variation on Yale database $b$ images

Lei Zhang and Dimitris Samaras [45] have suggested two novel methods for face recognition under 
subjectiveindefinite lighting by using spherical harmonics illumination representation, which need only one training image per subject and no 3D shape information. Their methods were centred on the recent result which established that the set of images of a convex Lambertian object attained under a wide variety of lighting conditions can be estimated accurately by a low-dimensional linear subspace. They delivered two methods to estimate the spherical harmonic basis images spanning their space from just one image.

Chengjun Liu [46] has offered a novel pattern recognition framework by exploiting on dimensionality increasing techniques. In specific, the framework incorporates Gabor image representation, a novel multiclass Kernel Fisher Analysis (KFA) method, and fractional power polynomial models for enlightening pattern recognition performance. Gabor image representation, which increases dimensionality by integrating Gabor filters with different scales and orientations, was regarded as by spatial frequency, spatial locality, and orientational discernment for coping with image variabilities such as illumination variations. The KFA method first performs nonlinear mapping from the input space to a high-dimensional feature space, and then outfits the multiclass Fisher discriminant analysis in the feature space.

Xudong Xie and Kin-Man Lam [47] have suggested an efficient representation method unaffected to varying illumination is proposed for human face recognition. Theoretical analysis based on the human face model and the illumination model shows that the effects of varying lighting on a human face image can be demonstrated by a sequence of multiplicative and additive noises. Instead of work out thesenoises, which was very difficult for real applications, they aim to reduce or even remove their effect. In their method, a local standardization technique was applied to an image, which could effectively and efficiently eradicate the effect of uneven illuminations while keeping the local geometric properties of the sort out image the same as in the corresponding image under normal lighting condition. After giving out, the image under varying illumination will have similar pixel values to the corresponding image that is under normal lighting condition. Then, the face recognition used exercised images.

Yasufumi Suzuki and Tadashi Shibata [48] have recommended that a pseudo-2D Hidden Markov Model-based face identification system retaining the edge-based feature representation has been developed. In the HMM-based face recognition algorithms, 2D discrete cosine transform (DCT) is frequently used for generating feature vectors. But, DCT-based feature representations were not forceful against the variation in illumination changes. Appropriate to enhance the robustness against illumination conditions, the edge-based feature representation has been engaged. Their edge-based feature representation has previously been applied to robust face detection in our previous work and was well-suited to processing in the committed VLSI hardware system which they have developed for real-time performance.

Shaohua Kevin Zhou et al. [49] have defined that the out-dated photometric stereo algorithms pay a Lambertian reflectance model with a varying albedo field and involve the presence of only one object. In their paper, they take a broad view photometric stereo algorithm to handle all appearances of all objects in a class, in particular the human face class, by making use of the linear Lambertian property. A linear Lambertian object was one which has linearly extend over by a set of basis objects and has a Lambertian surface. The linear property leads to a rank constraint and, ensuring, a factorization of an observation matrix that consists of prototype images of different objects (e.g., faces of different subjects) under different, unknown illuminations.

Stan Z. Li et al. [50] have offered a novel solution for illumination invariant face recognition for indoor, cooperative-user applications. First, they furnished an active near infrared (NIR) imaging system that was able to produce face images of good condition irrespective of visible lights in the surroundings. Second, they demonstrated that the resultant face images encode essential information of the face, subject only to a monotonic transform in the gray tone; based on those, they use local binary pattern (LBP) features to reimburse for the monotonic transform, thus developing an illumination invariant face representation.

Mario I. Chacon et al. [51] have offered a new method intended to design a fuzzy face recognition system. Face feature lines, new features proposed in the paper, were merged in the feature vector used to design the pattern recognition system. Face feature lines were deliberated as new features based on erstwhile studies related to face recognition tasks on newborns. Moreover the face feature lines the feature vector integrates eigenvectors of the face image obtained with the Karhunen-Loeve transformation. The fuzzy face recognition system was based on the Gath-Gheva fuzzy clustering method and the Abonyi and Szeifert classification system.

Xuan Zou et al. [44] have offered that the illumination variation problem was one of the familiar problems in face recognition in uncontrolled environment. In their paper an wide-ranging and current survey of the existing techniques to address the problem was presented. Their survey covers the submissive techniques that attempt to solve the illumination problem by studying the visible light images in which face appearance has been transformed by varying illumination, as well as the active techniques that aim to acquire images of face modalities invariant to surrounding illumination.

\section{Review Based on Occlusion}

Occlusion is a mutual difficulty comes across in applications of automatic face recognition. Causes of occlusion include apparel such as eyeglasses, sunglasses, hats, or scarves, in addition to objects such as cell phones placed in front of the face. Also, even in the absence of an occluding object, violations of an assumed model for face appearance may act like occlusions: e.g., shadows due to extreme illumination violate the assumption of a low dimensional linear illumination model. Strength to occlusion is so essential to practical face recognition. A number of occluded face recognition methods have been proposed in the past years. The figure 5 shows some of the occluded images in the AR face database. 


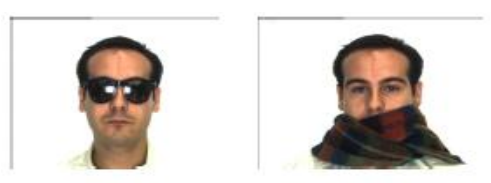

Fig. 5. AR database images based on the occlusion

Aleix M. Martõ̂̂nez [52] has recommended that the classical way of bidding to solve the face (or object) recognition problem was by using large and representative data sets. In their involvement, they describe a probabilistic approach that was able to reimburse for loosely localized, partially occluded, and expression-variant faces even when only one single training sample per class was available to the system. To solve the localization problem, they find the subspace (within the feature space, e.g., eigenspace) that represents their error for each of the training images. To resolve the occlusion problem, each face was divided into $\mathrm{k}$ local regions which are analyzed in isolation. In contrast with other methods where a simple voting space is used, they present a probabilistic method that analyzes how good a local match was.

Tae Young Kim et al. [53] have suggested that the Subspace analysis such as the principal component analysis (PCA) and linear discriminant analysis (LDA) were commonly used feature extraction methods for face recognition. They intended a new method that uses the twodimensional principal component analysis (2D PCA) for occlusion invariant face recognition. In divergence to $1 \mathrm{D}$ PCA, 2D PCA projects a 2D image directly onto the 2D PCA subspace, and each row of the resulting feature matrix exhibits the distribution of corresponding row of the image. And so by classifying each row of the feature matrix independently, they can easily identify the locally occluded parts in a face image. The occlusion invariant face recognition algorithm consists of two parts: occlusion detection and partial matching. To detect occluded regions, they apply a novel combined k-NN and 1-NN classifier to each row of the feature matrix of the test face.

Dahua Lin and Xiaoou Tang [54] have offered that a outline to automatically detect and recover the occluded facial region. They first develop a Bayesian formulation combining the occlusion detection and recovery stages. Formerly a feature valuation model is developed to drive both the detection and recovery processes, which captures the face priors in both global correlation and local patterns. Based on their formulation, they further propose graph cut-based detection and confidence- oriented sampling to reach optimal detection and recovery respectively. Correlated to traditional works in image repairing, their approach was separate in three aspects: (1) it frees the user from marking the occlusion area by incorporating an automatic occlusion detector; (2) it learns a face quality model as a criterion to guide the whole procedure; (3) it couples the detection and occlusion stages to all together achieve two goals: exact occlusion detection and high quality recovery.

Wen Gao et al. [55] have labelled that the gaining and substances of a large-scale Chinese face database: the CAS-PEAL face database. We discuss the assessment procedure based on the CAS-PEAL-R1 database and present the performance of four algorithms as a baseline to do the following: 1) elementarily evaluate the difficulty of the database for face recognition algorithms; 2) preference evaluation results for researchers using the database; and 3) identify the strengths and weaknesses of the commonly used algorithms.

Hyun Jun Oh et al. [56] have projected a novel occlusion invariant face recognition algorithm based on selective local non-negative matrix factorization (S-LNMF) method. The proposed algorithm was collected of two phases; the occlusion recognition phase and the selective LNMF-based recognition phase. They used a local approach to effectively detect partial occlusions in an input face image. A face image was first divided into a finite number of disjointed local patches, and then each patch was represented by PCA (principal component analysis), obtained by consequent occlusion-free patches of training images. And the 1-NN threshold classifier was used for occlusion detection for each patch in the corresponding PCA space. In the recognition phase, by employing the LNMF-based face representation, they wholly use the LNMF bases of occlusion-free image patches for face recognition. Euclidean nearest neighbour rule was applied for the matching.

Kazuhiro Hotta [57] has offered the use of Support Vector Machine (SVM) with local Gaussian summation kernel for tough face recognition under partial occlusion. The efficiency of SVM and local features has been reported. Since conventional methods apply one kernel to global features and global features were inclined easily by noise or occlusion, the conventional methods are not robust to occlusion. The recognition method based on local features, was robust to occlusion because partial occlusion affects only specific local features. Consecutively to utilize their property of local features in SVM, local kernels were applied to local features. The use of local kernels in SVM requires local kernel integration. The abstract of local kernels was used as the integration method. The efficiency and sturdiness of their method are shown by comparison with global kernel based SVM.

Hyun Jun Oh et al. [58] have projected a novel occlusion invariant face recognition algorithm based on selective local nonnegative matrix factorization (S-LNMF) method. The algorithm was collected of two phases; the occlusion detection phase and the selective LNMF-based recognition phase. They used local approach to efficiently detect partial occlusion in the input face image. A face image was first divided into a bounded number of disorganized local patches, and then each patch was represented by PCA (Principal Component Analysis), obtained by resultant occlusion-free patches of training images. And 1-NN threshold classifier was used for occlusion detection for each patch in the corresponding PCA space. In the identification phase, by employing the LNMF-based face representation, they utterly use the LNMF bases of occlusion- free image patches for face recognition. Euclidean nearest neighbour rule was applied for the matching.

Xiaoyang Tan et al. [59] have described that the Recognition in hysterical situations was one of the most imperative bottlenecks for practical face recognition systems. In particular, few researchers have addressed the confront to 
identify non-cooperative or even perverse subjects who try to cheat the recognition system by intentionally changing their facial appearance through such tricks as variant expressions or disguise (e.g. by partial occlusions). Their paper addresses these problems within the framework of resemblance matching. A novel awareness inspired non-metric partial similarity measure has introduced, which was potentially useful in deal with the concerned problems because it can help capturing the major partial similarities that were leading in human insight. Two methods, based on the general golden section rule and the maximum margin criterion, respectively, were proposed to automatically set the resemblance entry.

Kelsey Ramirez-Gutierrez et al. [60] have offered a face recognition algorithm based on histogram equalization methods. Those methods allow standardizing the faces illumination dropping in such way the variations for further features extraction; which were extracted using the image phase spectrum of the histogram steady image together with the principal components analysis. Their scheme allows a decline of the amount of data without much information loss. Evaluation results show that their feature extraction system, when used together with the support vector machine (SVM), provides a recognition rate higher than $97 \%$ and a verification error lower than $0.003 \%$.

\section{Review Based on Other Researches}

Shaohua Kevin Zhou et al. [61] have offered an image-based method for face recognition across different illuminations and poses, where the term image-based means that no precise prior three-dimensional models are needed. As face recognition under illumination and pose variations involves three factors namely: identity, illumination, and pose, generalizations in all those three factors are required. Those existing recognition advance that is able to simplify in the identity and illumination dimensions and handle a given set of poses. Explicitly, their approach derives an identity signature that was illumination- and pose-invariant, where the character was tackled by means of subspace encoding, the illumination was regarded as with a Lambertian reflectance model, and the given set of poses was treated as a whole.

Tarrés et al [62] have offered a new method for face recognition that can handle with partial occlusion or strong variations in facial expression. Their methods try to solve the face recognition problem from a near holistic perspective. The main idea was to "eliminate" some type which may cause a reduction of the recognition exactness under occlusion or expression changing. To test and evaluate the routine of the new technique, a series of experiments have been conceded out which have shown improved performance and robustness when compared to the classical PCA.

Xiujuan Chai et al. [63] have described that the Pose and illumination changes from picture to picture were two main barriers toward full automatic face recognition. In their paper, a novel method to handle both pose and lighting condition concurrently was proposed. First, some located facial landmarks and a priori statistical deformable 3D model are used to recover a detailed 3D shape. Based on the improved 3D shape, the "texture image" calibrated to a standard illumination is generated by spherical harmonics ratio image and finally the illumination independent $3 \mathrm{D}$ face is reconstructed completely. Their method combines the strength of statistical deformable model to describe the shape information and the compact representations of the illumination in spherical frequency space, and handle both the pose and illumination variation all together.

Dibeklioglu et al. [64] have projected that the Automatic localization of 3D facial features was important for face recognition, tracking, modeling and expression analysis. Methods urbanized for 2D images were shown to have problems working across databases acquired with different illumination conditions. Expression variations, pose variations and occlusions also obstruct accurate detection of landmarks. In their paper they measure a fully automatic 3D facial landmarking algorithm that relies on accurate statistical modeling of facial features. Their algorithm can be engaged to model any facial landmark, provided that the facial poses present in the training and test conditions are similar. They test their algorithm on the recently acquired Bosphorus 3D face database, and also examine cross-database performance by using the FRGC database.

Kailash J. Karande et al. [4] have addressed that the difficulty of face recognition under variation of illumination and poses with large rotation angles using independent component analysis (ICA). Face recognition using ICA, based on information theory concepts, look for a computational model that best describes face, by extracting most appropriate information contained in that face. ICA approach used there to extract global features seems to be an sufficient method due to its simplicity, speed and learning capability. The pre-processing was done by principle component analysis (PCA) before applying the ICA algorithm for training of images. The independent components obtained by ICA algorithm are used as feature vectors for classification. The Euclidian distance classifier was used for testing of the images.

Kavitha R. Singh et al. [65] have described that the Face recognition being the most important biometric peculiarity it still faces many challenges, like pose variation, illumination variation etc. When such variations were present in both pose and illumination, all the algorithms were seriously exaggerated by these variations and their performance gets tainted. In their paper, they were presenting a detail survey on 2D face recognition under such uncontrolled conditions. They have explored different techniques proposed for illumination and pose problem in addition with the classifiers that have been successfully used for face recognition in wide-ranging.

\section{CONCLUSION}

Face recognition has been considered for over two decades, and it is still an active subject owing to extensive practical applications. Several potential applications include law enforcement, access control, security surveillance and monitoring, bankcard identification, and human-robot interface. In all these cases, face recognition acts as a critical role. It is a difficult prototype recognition problem due to the complex pattern distributions from large variations in facial expressions, facial details, and illumination conditions. In this paper, we have offered an extensive review of the 
significant researches in existence for face recognition based on various conditions such as pose, illumination, occlusion and expression. A concise description about the face recognition methods is also presented. The object of this research survey is to help the budding researchers in the field of content based video indexing and retrieval to understand the available methods and to support their further research.

\section{REFERENCES}

[1] H. S. Lee and D. Kim, "Generating frontal view face image for pose invariant face recognition," Pattern Recognition Letters, Vol.27, pp.747-754, 2006.

[2] C.H. Hoi and M.R.Lyu, "Robust face recognition using minimax probability machine," In Proc. IEEE Conf. on Multimedia and Expo (ICME'04), pp. 1175-1178, 2004.

[3] H. Zhang, Y. Li, L. Wang, and C. Wang, "Pose insensitive face recognition using feature transformation," IJCSNS International Journal of Computer Science and Network Security, Vol.7 No.2, February 2007.

[4] X.N Song, . Zheng, X. Wu, X. Yanga, and J. Yang, "A complete fuzzy discriminant analysis approach for face recognition," Applied Soft Computing, Vol. 10, No. 1, pp. 208-214, Jan 2010.

[5] A.K. Jain, A. Ross, and S.Prabhakar, "An introduction to biometric recognition," IEEE Transactions On Circuits And Systems For Video Technology, Vol. 14, No. 1, Jan. 2004.

[6] A. Jain, L. Hong, and S. Pankanti, "Biometric identification," Communications of the ACM, Vol. 43, No. 2, Feb. 2000.

[7] Z. Zhu and Q. Ji, "Robust pose invariant facial feature detection and tracking in real-time," In proc. of the 18th International Conference on Pattern Recognition, pp.1092-1095, 2006.

[8] K. J. Karande and S. N.Talbar, "Face recognition under variation of pose and illumination using independent component analysis," International Journal on Graphics, Vision and Image Processing (GVIP), Vol. 8, No.4, December 2008.

[9] W. Zhao, R.Chellappa, P. J. Phillips, and A. Rosenfeld, "Face recognition: a literature survey", ACM Computing Survey, pp. 399-458, Dec 2003

[10] S. Lin, "An introduction to face recognition technology," Informing Science Special Issue on Multimedia Informing Technologies, Vol.3, No. 1, 2000.

[11] G. Shakhnarovich and B. Moghaddam, "Face recognition in subspaces," Springer, Heidelberg, May 2004.

[12] F. Perronnin, J. L. Dugelay, and K. Rose, "A probabilistic model of face mapping with local transformations and its application to person recognition," IEEE Transactions on Pattern Analysis and Machine Intelligence, Vol. 27, No. 7, July 2005.

[13] R. Chellappa, C. Wilson, and S. Sirohey, "Human and machine recognition of faces: a survey," In Proc. of the IEEE conference, Vol. 83, No. 5, pp. 705-740, May 1995.

[14] N.V.S.L. Swarupa and D.Supriya, "Face recognition system," International Journal of Computer Applications, Vol. 1,No. 29,pp.36-41,2010.

[15] S. T. Gandhe, K. T. Talele, and A.G.Keskar, "Face recognition using contour matching," IAENG International Journal of Computer Science, Vol.35, No.2, 2008.

[16] L.Wiskott, J. M. Fellous, N. Kruger, and C. V. Malsburg, "Face recognition by elastic graph matching," IEEE Transactions on PAMI, Vol.19, no. 7, 1997.

[17] Z. Zhang, "Feature-based facial expression recognition: experiments with a multi-layer perceptron," In Technical Report INRIA, 3354, 1998

[18] X. Wei, Z. Zhu, L. Yin, and Q. Ji, "A real-time face tracking and animation system," In Proc. of the IEEE Workshop on FPIV, 2004.

[19] H. K. Ekenel and B. Sankur, "Multiresolution face recognition," Image and Vision Computing, Vol.23, pp.469-477, 2005.

[20] A. Eleyan, H. S. Ozkaramanli, and H. Demirel, "Complex wavelet transform-based face recognition," EURASIP Journal on Advances in Signal Processing, Vol. 2008, pp.1-13, 2008.

[21] Y. Moses, Y. Adini, and S. Ullman, "Face recognition: The problem of compensating for changes in illumination direction," IEEE Transactions on Pattern Analysis and Machine Intelligence, Vol.19, No.7, pp.721-732, 1997.

[22] S. Amnon and R. Tammy, "The quotient image: class based re-rendering and recognition with varying illuminations", IEEE Transaction on Pattern Analysis and Machine Intelligent, Vol.23, No.2, pp.129-139, 2001.
[23] V. Blanz and T. Vetter, "Face recognition based on fitting a $3 \mathrm{~d}$ morphable model," IEEE Transactions on Pattern Analysis and Machine Intelligence, Vol. 25, No. 9, Sep. 2003.

[24] H. Wang, Y. Wang, and Y. Cao, "Video-based face recognition: a survey," World Academy of Science, Engineering and Technology, Vol.60, pp. 293-302,2009.

[25] Z. Zhou, J. H. Fu, H. Zhang, and Z. Chen, "Neural network ensemble based view invariant face recognition", J. Comput. Study Develop., Vol. 38, no. 9, pp. 1061-1065, 2001.

[26] X. Zou, Kittler, J., Messer, K.Surrey Univ, and Guildford, "Illumination invariant face recognition: a survey," In Proc. of the First IEEE International Conference on Biometrics: Theory, Applications, and Systems (BTAS 2007), crystal city, VA, pp.1-8, 2007.

[27] A. Pentland, B. Moghaddam, and T. Starner, "View-based and modular eigenspace for face recognition," In Proc. of the IEEE Conf. Comput. Vision Pattern Recognition, pp. 84-91, 1994.

[28] M. Turk and A. Pentland, "Eigenfaces for recognition," J. Cogn Neurosci., Vol. 13, No. 1,pp. 71-86, 1991.

[29] L. Shen, Z. Ji, L. Bai, and C. Xu, "DWT based HMM for face recognition," Journal of Electronics, Vol. 24, No. 6, pp. 835-837, 2007.

[30] B.S.Manjunath, R. Chellappa, and C. von der Malsburg, "A feature based approach to face recognition," In Proc. of the IEEE Computer Society Conference on Computer Vision and Pattern Recognition (CVPR '92), pp.373-378, June 1992.

[31] K.J. Priya and R.S.Rajesh, "Local statistical features of dual tree complex wavelet transform on parallelogram image structure for face recognition with single sample," In proc. of the International Conference on Recent Trends in Information, Telecommunication and Computing (ITC),pp.50-54, May 2010.

[32] S. Kim, S. T. Chung, S. Jung, and S. Cho, "An improved illumination normalization based on anisotropic smoothing for face recognition," International Journal of Computer Science and Engineering, Vol. 2, No.3,pp.89-95,2008.

[33] A.J. O'Toole, P. J. Phillips, F.Jiang, J. Ayyad, N. Penard, and H. Abdi, "Face recognition algorithms surpass humans matching faces over changes in illumination," IEEE Transactions on Pattern Analysis and Machine Intelligence, Vol. 29, No. 9, pp. 1642-1646, June 2007.

[34] H. Wechsler, P. Phillips, V. Bruce, F. Soulie, and T. Huang, "Face recognition: from theory to applications," Springer-Verlag, 1996.

[35] S. Gong, S.J. Mckenna, and A. Psarrou, "Dynamic vision: from images to face recognition", Imperial College Press and World Scientific Publishing, 2000.

[36] T. Shan, B. C. Lovell, and S. Chen, "Face recognition robust to head pose from one sample image," In Proc. of the 18th International Conference on Pattern Recognition, (ICPR 2006), pp.515-518, 2006.

[37] X. Zhang and Y. Gao, "Face recognition across pose: A review," Pattern Recognition, Vol. 42, No. 11, pp. 2876-2896, 2009.

[38] T. K. Kim and J.Kittler, "Design and fusion of pose-invariant face-identification experts," IEEE Transactions On Circuits And Systems For Video Technology, Vol. 16, No. 9, pp. 1096-1106, September 2006

[39] H. F.Ng, "Pose-invariant face recognition security system," Asian Journal of Health and Information Sciences, Vol. 1, No. 1, pp. 101-111, 2006.

[40] H. Eidenberger, "Kalman filtering for pose-invariant face recognition," In Proc. of the IEEE International Conference on Image Processing, Atlanda,pp.2037-2040, 2006.

[41] X. Chai, S. Shan, X. Chen, and W. Gao, "Locally linear regression for pose-invariant face recognition," IEEE Transactions On Image Processing, Vol. 16, No. 7, pp.1716-1725, July 2007.

[42] R. Singh, M. Vatsa, Arun Ross, and Afzel Noore, "A mosaicing scheme for pose-invariant face recognition," IEEE Transactions On Systems, Man, And Cybernetics-Part B: Cybernetics, Vol. 37, No. 5, pp.1212-1225, October 2007

[43] H. Demirel, and G. Anbarjafari, "Pose invariant face recognition using probability distribution functions in different color channels," IEEE Signal Processing Letters, Vol. 15, pp,537-540, 2008.

[44] S. J.D. Prince, and J. H. Elder, "Tied factor analysis for face recognition across large pose differences," IEEE Transaction on Pattern Analysis and Machine Intelligence, Vol. 30, No.6, pp.970-984, 2008.

[45] L. Zhang and Dimitris Samaras, "Face recognition from a single training image under arbitrary unknown lighting using spherical harmonics," IEEE Transactions On Pattern Analysis And Machine Intelligence, Vol. 28, No. 3, pp. 351-363, March 2006.

[46] C. Liu, "Capitalize on dimensionality increasing techniques for improving face recognition grand challenge performance," IEEE 
Transaction on Pattern Analysis and Macchine Intelligence, Vol.28, No.5, pp.725-737,May 2006.

[47] X. Xie and Kin-Man Lam, "An efficient illumination normalization method for face recognition," Pattern Recognition Letters, Vol.27, No.6, pp.609-617, 2006.

[48] Y. Suzuki and T. Shibata, "Illumination-invariant face identification using edge-based feature vectors in pseudo-2d hidden markov models," In Proc. of 14thEuropean Signal Processing Conference (EUSIPCO 2006), Florence, Italy, pp. 1-5, Sep.2006.

[49] S. K. Zhou, Gaurav Aggarwal, Rama Chellappa, and David W. Jacobs, "Appearance characterization of linear lambertian objects, generalized photometric stereo, and illumination-invariant face recognition," IEEE Transactions On Pattern Analysis And Machine Intelligence, Vol. 29, No. 2, pp.230-245, Feb. 2007.

[50] S. Z. Li, R. F. Chu, S. C. Liao, and L. Zhang, "Illumination invariant face recognition using near-infrared images," IEEE Transactions $O n$ Pattern Analysis And Machine Intelligence, Vol. 29, No. 4, pp. 1-13, April 2007.

[51] M. I. Chacon, P.P. Rivas, and A.G. Ramirez, "A fuzzy clustering approach for face recognition based on face feature lines and eigenvectors," Engineering Letters, Vol.15, No.1, pp.1-10, 2007.

[52] A. M. MartôÂnez, "Recognizing imprecisely localized, partially occluded, and expression variant faces from a single sample per class,' IEEE Transaction on Pattern Analysis and Machine Intelligence, Vol.24, No.6, pp.748-763, 2002.

[53] T. Y. Kim, K. M. Lee, S. U. Lee, and C.H. Yim, "Occlusion invariant face recognition using two-dimensional PCA," Advances In Computer Graphics and Computer Vision, Vol.4, No. 8, pp.305-315,2007.

[54] D. Lin and X. Tang, "Quality-driven face occlusion detection and recovery," In Proc. of the IEEE Conference on Computer Vision and Pattern Recognition, pp.1-7, 2007.

[55] W. Gao, B.Cao, S. Shan, X. Chen, D. Zhou, X. Zhang, and D. Zhao, "The CAS-PEAL large-scale chinese face database and baseline evaluations," IEEE Transaction on Systems, Man, and Cybernetics-Part a: Systems and Humans, Vol. 38, No.1, pp. $149-161,2008$.

[56] H. J. Oh, K. M. Lee, and S. U. Lee, "Occlusion invariant face recognition using selective local non-negative matrix factorization basis images," Image and Vision Computing, Vol.26,No.11,pp.1515-1523,2008.

[57] K. Hotta, "Robust face recognition under partial occlusion based on support vector machine with local Gaussian summation kernel," Image and Vision Computing, Vol.26, No. 11, pp.1490-1498,2008.

[58] H. J. Oh, K. M. Lee, S. U. Lee, and C.H. Yim, "Occlusion invariant face recognition using selective lnmf basis images," Computer Vision, Vol.3851,pp.120-129,2009

[59] X. Tan, S. Chen, Z.H. Zhou, and J. Liu, "Face recognition under occlusions and variant expressions with partial similarity," IEEE Tracsactions on Information Forensics and security, Vol.4, No.2, pp.1-13, 2009.
[60] K. R. Gutierrez, D. C. Perez, J. O. Mercado, M. N. Miyatake, and Hector Perez-Meana, "A face recognition algorithm using eigen phases and histogram equalization," International journal of Computers, Vol. 5, No.1,pp.34-41,2011.

[61] S. K. Zhou and R. Chellappa, "Image-based face recognition under illumination and pose variations," J. Opt. Soc. Am. A, Vol. 22, No. 2, pp. 217-229, February 2005.

[62] F. Tarrés, A. Rama, and L. Torres, "A novel method for face recognition under partial occlusion or facial expression variations," In Proc. of the 47th International Symposium (ELMAR-2005) on Multimedia Systems and Applications, Zadar, Croatia, June 2005.

[63] X. Chai, L. Qing, S. Shan, X. Chen, and W. Gao, "Pose invariant face recognition under arbitrary illumination based on $3 \mathrm{~d}$ face reconstruction," Audio- and Video-Based Biometric Person Authentication, Vol.3546,pp.956-965,2005.

[64] H. Dibeklioglu, , A. A. Salah, and L. Akarun, "3D facial landmarking under expression, pose, and occlusion variations," In Proc. of the IEEE International Conference on Biometrics Theroy, Applications and Systems, Arlington, pp.1-6, 2008.

[65] K. R. Singh, M. A. Zaveri, and M. M.Raghuwanshi, "Illumination and pose invariant face recognition: a technical review," International Journal of Computer Information Systems and Industrial Application (IJCISIM), Vol.2, pp.29-38, 2010.

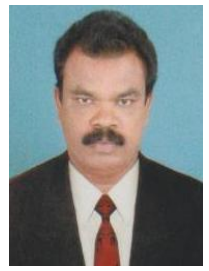

S.Muruganantham received the M.C.A degree in Manonmaniam Sundaranar University, Tamilnadu, India, in 1996. The M.Tech Degree in Manonmaniam Sundaranar University Tamilnadu, India, in 2004. In 1997 he joined the M.C.A Department, S.T.Hindu college, Tamilnadu, India as an Assistant Professor. His research interest includes Image processing, Operating System, Software Engineering.

He has published international journals. He is currently a research scholar in the area of Face recognition. He is Life member of CSI, ACCS, IAENG, ISTE and IACSIT.

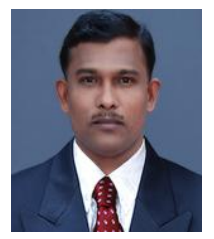

Dr. T. Jebarajan received the B.E Degree in Computer Science and Engineering in National Engineering College, Tamilnadu, India. The M.E. Degree in Computer Scinece and Engineering in Jadavpur University, Calcutta. The Ph.D in Computer science And Engineering from Manonmaniam Sundaranar University, Tamilnadu, India.

He has worked as H.O.D. of Computer science and Engineering Department in Noorul Islam Deemed University, Kanyakumari District, Tamilnadu, India more than 15 years. Now he is working as a principal of KINGS College of Engineering, Chennai, Tamilnadu, India. He has National and International publication on the field of Computer Science. 